\title{
Signaling through CD28 and CTLA-4 controls two distinct forms of $T$ cell anergy
}

\author{
Andrew D. Wells, ${ }^{1}$ Matthew C. Walsh, ${ }^{1}$ Jeffrey A. Bluestone, ${ }^{2}$ and Laurence A. Turka ${ }^{1}$ \\ ${ }^{1}$ Department of Medicine, University of Pennsylvania, Philadelphia, Pennsylvania, USA \\ ${ }^{2}$ UCSF Diabetes Center, University of California, San Francisco, San Francisco, California, USA
}

Address correspondence to: Andrew D. Wells or Laurence A. Turka, University of Pennsylvania, 700 Clinical Research Building, 415 Curie Boulevard, Philadelphia, Pennsylvania 19104-6144, USA.

Phone: (215) 898-1018; Fax: (215) 573-2880; E-mail: adwells@mail.med.upenn.edu or turka@mail.med.upenn.edu.

Received for publication May 8, 2001, and accepted in revised form August 8, 2001.

\begin{abstract}
Primary T cell proliferative responses to TCR ligation plus CD28 costimulation are surprisingly heterogeneous. Many cells that enter G1 fail to progress further through the cell cycle, and some of these cells subsequently fail to divide upon restimulation, even in the presence of IL-2. Such IL-2-refractory anergy is distinct from the IL-2-reversible anergy induced by TCR occupancy in the absence of CD28 costimulation. Here, we focus on the contributions of cell cycle progression and costimulatory (CD28/CTLA-4) signals in the regulation of anergy. We show that CD28 costimulation is not sufficient for anergy avoidance and that activated $T$ cells must progress through the cell cycle in order to escape anergy. Induction of this "division-arrest" form of anergy requires CTLA-4 signaling during the primary response. Also, cell division per se is not sufficient for anergy avoidance: the few $\mathrm{T}$ cells that undergo multiple rounds of cell division during overt CD28 costimulatory blockade do not escape the ultimate induction of clonal anergy. Anergy avoidance by primary $\mathrm{T}$ cells is thus a multistep process: in order to participate in a productive immune response, an individual $T$ cell activated through its antigen receptor must receive CD28 costimulation and progress through the cell cycle. Anergy may be induced either through a combination of CTLA-4 signaling and the failure of cell cycle progression, or through a proliferation-independent mechanism in which TCR ligation occurs in the absence of CD28.
\end{abstract}

J. Clin. Invest. 108:895-904 (2001). DOI:10.1172/JCI200113220.

\section{Introduction}

Using techniques to analyze functional effector responses of single cells, a number of investigators have shown that the behavior of a population of optimally activated $T$ cells is surprisingly heterogeneous. Weaver, Bucy, and colleagues were among the first to show this, using immunohistochemical approaches to detect cytokine production at the individual cell level (1). Using a technique based on the fluorescent dye CFSE, we subsequently identified a large degree of proliferative heterogeneity in both polyclonal and monoclonal populations of activated T cells $(2,3)$. For instance, up to $35 \%$ of $\mathrm{T}$ cells optimally activated through TCR/CD3 and CD28 fail to divide, despite induction of G1-associated genes such as CD69 and CD25 $(2,3)$. More recently, we have studied the consequences of this proliferative heterogeneity (4). These investigations revealed that cells that fail to divide in response to primary stimulation (a population that we term "primary nonproliferative T cells") do not divide upon secondary stimulation. This state of anergy is novel, as unlike classic clonal anergy induced by TCR/CD3 ligation without $\mathrm{CD} 28$ costimulation, anergy occurring in primary nonproliferative $T$ cells cannot be reversed by IL- 2 .

These studies indicate that primary $\mathrm{T}$ cells are susceptible to two distinct types of anergy induction when stimulated. The current studies were undertaken to identify the mechanisms and parameters that regulate the induction of various forms of anergy. In particular, we chose to focus on CD28/CTLA-4 signals and on cell division. $T$ cell receptor-coupled mitogenic signals are amplified by signals transduced through CD28 and are opposed by signals transduced through CTLA- $4(5,6)$. The balance between these opposing signals in part determines the eventual fate of the responding $T$ cells; CD28 promotes IL-2 production, clonal expansion, anergy avoidance, and effector function $(7,8)$, whereas CTLA-4 signals result in abortive $T$ cell activation, poor IL-2 production, and anergy (9-14). It has been proposed that the ability of CD28 to prevent anergy (and conversely, of CTLA-4 signaling to induce it) is a secondary consequence of its ability to regulate $\mathrm{T}$ cell proliferation (15-18). This implies that avoidance (or induction) of anergy depends on the dilution (or accumulation) of an anergy factor(s) that is rapidly synthesized after TCR stimulation and whose subsequent intracellular concentration is regulated by cell division and dilution $(15,19)$. For instance, CD28 costimulation during primary activation may promote anergy avoidance and subsequent $T$ cell effector function as a consequence of its ability to support multiple rounds of cell division $(2,20)$. Conversely, signals through CTLA-4 may promote anergy and negatively influence $\mathrm{T}$ cell effector function by inhibiting cell-cycle progres- 
sion during the primary response. Alternatively, CD28 and CTLA-4 may directly regulate anergy and cell fate, independent of cell proliferation. These two possibilities are not mutually exclusive, and this area has remained unresolved and controversial.

To investigate the potentially separate roles of proliferation and CD28/CTLA-4 signals in anergy induction, we blocked B7-CD28 and/or B7-CTLA-4 interactions during primary $\mathrm{T}$ cell activation, purified the primed $\mathrm{T}$ cells based on the number of cell divisions they achieved, and assessed their responsiveness to secondary TCR-, CD28-, and IL-2-mediated signals. Using this approach, we find that two distinct forms of anergy can be resolved in a population of $\mathrm{CD} 4^{+} \mathrm{T}$ cells stimulated in vitro. One form results from TCR ligation in the absence of CD28 costimulation. This form of anergy is reversible by IL-2, but cannot be prevented by progressing through the cell cycle, indicating that cell division alone is not sufficient for anergy avoidance in $\mathrm{T}$ cells. However, cell division appears to be necessary for anergy avoidance, as a second form of anergy results from the failure to proliferate after activation under conditions of sufficient CD28 costimulation. This form of anergy, which is refractory to IL-2, is controlled in part by signals transduced through the negative regulatory receptor CTLA- 4 . We conclude that anergy avoidance by primary $\mathrm{T}$ cells is a multistep process. Signals through CD28 and processes associated with cell division are together necessary and sufficient to prevent the induction of IL-2 reversible anergy. Furthermore, we suggest that CTLA-4 regulates anergy in two ways: the first is indirect, through inhibition of cell-cycle progression, and the second is through a specific signal that is required to induce IL-2 nonresponsive anergy among nonproliferating cells.

\section{Methods}

Mice, antibodies, and reagents. Pooled spleen and lymph node cells from female BALB/c mice, aged 8-16 weeks, were used for all experiments. mAb's against CD3 (1452C11), CD28 (37.51), and CTLA-4/CD154 (4F10) were purified from hybridoma supernatants. Anti-CTLA-4 Fab fragments were generated as described previously (10). CTLA4Ig was provided by R. Peach (Bristol-Meyers-Squibb, Seattle, Washington, USA). Purified, fluorochrome-conjugated $\mathrm{mAb}$ against $\mathrm{CD} 16 / \mathrm{CD} 32$ (FcBlock), Thy1.2, CD4, and IL-2 were purchased from PharMingen (San Diego, California, USA). mAb specific for p2 $7^{\text {kip } 1}$ was purchased from Transduction Laboratories (Lexington, Kentucky, USA). Rabbit antiserum against actin was purchased from Sigma Chemical Co. (St. Louis, Missouri, USA). CFSE (5- and 6-carboxyfluorescein diacetate succinimidyl ester) and TOPRO-3 were purchased from Molecular Probes Inc. (Eugene, Oregon, USA). Recombinant murine IL-2 was purchased from Genzyme Pharmaceuticals (Cambridge, Massachusetts, USA), and was used at 10-50 U/ml.

Cell labeling and culture conditions. Cell isolation and fluorescent labeling of cells with CFSE was performed as described previously (2). Briefly, pooled spleen and lymph node cells were incubated with CFSE in PBS at a final concentration of $2 \mu \mathrm{M}$ for 3 minutes. CFSElabeled cells were stimulated with soluble anti-CD3 $\mathrm{mAb}(1 \mu \mathrm{g} / \mathrm{ml})$ at $2 \times 10^{6}$ to $4 \times 10^{6} / \mathrm{ml}$ in 24 -well plates. When indicated, cultures contained anti-CD28 mAb (1 $\mu \mathrm{g} / \mathrm{ml})$, CTLA4Ig $(10 \mu \mathrm{g} / \mathrm{ml})$, anti-CTLA-4 mAb (10 $\mu \mathrm{g} / \mathrm{ml})$, or anti-CTLA-4 Fab $(20 \mu \mathrm{g} / \mathrm{ml})$. For primary cultures, the cells were stimulated for 4 days, washed and replated in fresh medium for 48 hours, and then sorted based on CFSE fluorescence. Sorted T cells were restimulated in 96-well round-bottom plates with irradiated syngeneic splenocytes (fourfold excess) and soluble anti-CD3 $(1 \mu \mathrm{g} / \mathrm{ml})$ in the presence or absence of IL-2 $(10 \mathrm{U} / \mathrm{ml})$ for 4 days. Secondary proliferation (CFSE fluorescence) was assessed by flow cytometry.

Flow cytometry and cell sorting. Cell-surface marker staining and flow cytometric analysis were performed on a FACSCalibur flow cytometer using CellQuest software (both, Becton Dickinson and Co., Franklin Lakes, New Jersey, USA). Methods using CFSE labeling to calculate the absolute number of mitotic events occurring in the culture have been described elsewhere (2). The vital dye TOPRO-3 was used to discriminate live and dead cells (2). Live, Thy $1.2^{+}$or $\mathrm{CD} 4^{+} \mathrm{T}$ cells were sorted based on CFSE fluorescence using a FACSVantage flow cytometer/sorter (Becton Dickinson and Co.), as described previously (4).

Intracellular cytokine staining. IL-2 production was assessed at the single-cell level as described previously (21), with some modifications. Cells that had been activated in primary culture for 4 days and rested for 48 hours were cultured for 5 hours with polystyrene beads coated with anti-CD3 and anti-CD28 mAb's $(5 \mu \mathrm{g} / \mathrm{ml}$ each; 5:1 bead/T cell ratio) in the presence of $2 \mu \mathrm{M}$ monensin (Sigma Chemical Co.). As primed T cells do not divide during this 5 -hour restimulation period (data not shown), cytokine production can be assessed as a function of primary proliferative history without the use of cell sorting.

Assessment of $p 27^{k i p 1}$ degradation. Pooled spleen and lymph node cells were cultured in 24-well plates $(4 \times$ $10^{6}$ cells $\left./ \mathrm{ml}\right)$ with soluble anti-CD3 $\mathrm{mAb}(1 \mu \mathrm{g} / \mathrm{ml})$ and anti-CD28 $\mathrm{mAb}(1 \mu \mathrm{g} / \mathrm{ml})$, CTLA4Ig $(10 \mu \mathrm{g} / \mathrm{ml})$, or anti-CTLA- $4 \mathrm{mAb}(10 \mu \mathrm{g} / \mathrm{ml})$. After 4 days, the cultures were washed and the cells were replated in fresh medium for 24 hours. Rested cells were then restimulated for 48 hours in 96 -well round bottom plates with polystyrene beads coated with anti-CD3 $(5 \mu \mathrm{g} / \mathrm{ml})$ in the presence or absence of IL-2 $(50 \mathrm{U} / \mathrm{ml})$. Whole-cell lysates were generated, subjected to SDS-PAGE $\left(3 \times 10^{5}\right.$ to $5 \times 10^{5}$ cell equivalents per lane), and transferred to nitrocellulose membranes. Blots were probed with mAb against $\mathrm{p} 27^{\mathrm{kip} 1}$ (1:1,000 dilution), and immunoreactive protein was visualized by autoradiography after probing with peroxidase-conjugated secondary antibody (Roche Molecular Biochemicals, Indianapolis, Indiana, USA) and incubation with a chemiluminescent reagent (Roche Molecular Biochemicals). Blots 
were stripped and reprobed with a rabbit anti-serum against actin $(1: 1,000)$ to control for loading.

\section{Results}

$C D 4^{+} T$ cells that proliferate in the absence of CD28 costimulation do not escape clonal anergy. The CD28 costimulatory molecule has a clear role in effector/memory $\mathrm{T}$ cell development (5) and in the avoidance of clonal anergy (15). However, previous studies have also suggested that both anergy avoidance $(4,16,18)$ and effector function $(3,22,23)$ in activated $\mathrm{CD}^{+} \mathrm{T}$ cell populations are tightly associated with cell division. It remains unclear whether the effects of CD28 or CTLA- 4 on the generation of an effector/memory pool are a direct consequence of signal transduction through these coreceptors, or are indirect, mediated through regulation of $\mathrm{T}$ cell proliferation.

To distinguish between these two possibilities, we modulated B7-mediated costimulatory signals during $T$ cell priming and assessed the ability of the resultant $\mathrm{CD}^{+} \mathrm{T}$ cells to produce IL-2 upon TCR re-ligation as a function of their primary proliferative behavior. For this model, bulk spleen and lymph node cells were labeled with CFSE and stimulated with anti-CD3 $\mathrm{mAb}$ in the presence of either control $\mathrm{mAb}$ or CTLA4Ig. In this system, antigen-presenting cells (APCs) present in the mononuclear cell preparation provide endogenous B7 costimulation to $\mathrm{T}$ cells, which is blocked by CTLA4Ig. After 4 days, the cells were washed and plated in fresh medium for 48 hours. Finally, $\mathrm{T}$ cells were restimulated for 5 hours by beads coated with antiCD3 and anti-CD28, and IL-2 production within the gated $\mathrm{CD}^{+}$subset was assessed by intracellular staining and flow cytometric analysis. As further cell division does not occur during this brief restimulation period, the CFSE profile of the cells at this time is representative of cell division during the primary response (ref. 3; data not shown). In the experiment shown in Figure 1, we confirm previous observations by our laboratory and others that the $T$ cell proliferative response under these conditions is heterogeneous. Even with B7 costimulation provided by APCs, up to $30 \%$ of the input precursor $\mathrm{T}$ cells failed to divide (ref. 2; Figure 1a), although virtually all cells (> 97\%) had received activating signals as assessed by induction of CD69 expression at 24 hours (ref. 2; data not shown). Blockade of costimulation by CTLA4Ig reduced the frequency of proliferative cells (ref. 2; Figure 1b). Upon rest and restimulation of these previously activated cells, we found that $48 \%$ of the divided $\mathrm{CD}^{+} \mathrm{T}$ cell pool primed in the presence of $\mathrm{B} 7 / \mathrm{CD} 28$ costimulation was able to produce IL-2 upon TCR/CD28 reengagement (Figure 1a, compare upper and lower left quadrants), whereas only $30 \%$ of the undivided $\mathrm{CD}^{+}{ }^{+} \mathrm{T}$ cell pool was able to do so (Figure 1a, compare upper and lower right quadrants). These data confirm the previously observed trend between cell division and IL-2 production at the single-cell level among $\mathrm{CD}^{+} \mathrm{T}$ cells derived from the same precursor pool $(3,4)$. Blockade of B7-mediated costimulation during primary stimulation using CTLA4Ig resulted in a marked reduction in both cell division and effector differentiation (Figure 1b), as only $15 \%$ of total $\mathrm{CD}^{+} \mathrm{T}$ cells were able to produce IL-2 upon TCR/CD28 reengagement (compared with 35\% in the cultures primed under physiological costimulatory conditions). However, a division-associated trend can also be observed under these conditions, as $25.5 \%$ of the rare proliferating $\mathrm{CD}^{+}{ }^{+} \mathrm{T}$ cells were able to synthesize IL-2, versus a frequency of only $13.7 \%$ within the much larger undivided pool (Figure 1b).

These results suggested to us the possibility that the rare population of $\mathrm{CD}^{+} \mathrm{T}$ cells that succeed in dividing after activation in the absence of B7-mediated costimulation might escape anergy induction. Bulk assays of anergy induction such as $\left[{ }^{3} \mathrm{H}\right]$ thymidine incorporation, which measure the proliferative response of all the T cells collectively, would be unable to detect the proliferative response of the few divided $\mathrm{CD}^{+} \mathrm{T}$ cells. To overcome this problem, CFSE-labeled $\mathrm{CD}^{+} \mathrm{T}$ cells that had been primed and rested as in Figure 1 were sorted into fractions representing cells that had either achieved two cell divisions or that had remained undivided throughout the entire culture period (Figure 2,
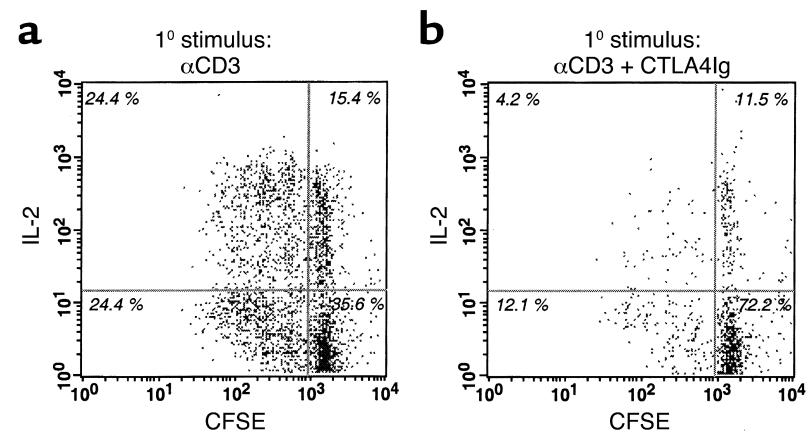

\section{Figure 1}

Regulation of T cell IL-2 production by both B7-mediated costimulation and cell division. (a) Pooled BALB/c lymph node and spleen cells were labeled with CFSE, and T cells were stimulated in the presence of endogenous B7-mediated costimulation by the addition of soluble anti-CD3 antibody $(1 \mu \mathrm{g} / \mathrm{ml})$. (b) Alternatively, B7-mediated costimulation was blocked during primary activation by the addition of CTLA4lg $(15 \mu \mathrm{g} / \mathrm{ml})$. After 4 days, cultures were washed, plated in fresh medium, and rested for an additional 48 hours. T cells were then restimulated for 5 hours by the addition of polystyrene beads $(5 \mu \mathrm{m})$ coated with anti-CD3 $(5 \mu \mathrm{g} / \mathrm{ml})$ and anti-CD28 $(5 \mu \mathrm{g} / \mathrm{ml})$ antibodies, and the frequency of CD4+ cells producing IL-2 was assessed by flow cytometry. Plots show IL-2 production as a function of cell division (CFSE fluorescence) in the $\mathrm{CD} 4^{+} \mathrm{T}$ cell subset. Vertical lines delineate the divided from the undivided cells, and horizontal lines denote the maximal fluorescence of cells stained with isotype control antibody. Values in each corner represent the proportion of the CD4 ${ }^{+}$ events that fall in each quadrant. Cells cultured in medium with no stimulus and stained with specific antibody were less than $0.1 \%$ positive for IL-2. These data are representative of three independent experiments. The frequency of IL-2 producers among CD3/CD28-primed T cells varied from experiment to experiment (range: 10-35\%); however, the frequency of IL-2 producers in cultures primed in the presence of CTLA4Ig was consistently reduced two- to fourfold. 
$a-n)$. These purified cells were then restimulated by the addition of soluble anti-CD3 antibody in the presence of syngeneic irradiated splenocytes, and proliferation was assessed at the single-cell level 96 hours later by CFSE intensity. As shown in Figure 2, a-n, we were able to obtain homogeneous populations of divided and undivided cells by cell sorting. Furthermore, this was a stable phenotype, as the level of CFSE staining was unaltered by continued culture in medium alone, indicating that the cells had ceased to proliferate during the rest period (Figure 2, b, c, i, and j).

Contrary to our prediction, both the divided and the undivided populations of $\mathrm{CD}^{+} \mathrm{T}$ cells from CTLA4Igtreated cultures were equally unresponsive to restimulation through TCR/CD28 as measured by proliferation (Figure 2, k and l; and Figure 2o, third set of columns). However, the proliferation of both populations of $\mathrm{CD}^{+} \mathrm{T}$ cells could be restored by the addition of IL-2 (Figure 2, $\mathrm{m}$ and n; and Figure 2o, fourth set of columns). This phenotype (i.e., a reduced capacity to produce IL-2 and proliferate in response to TCR/CD28 re-ligation, yet a relatively intact response to IL-2 provided exogenously) closely resembles anergy in $\mathrm{T}$ cell clones (15). Therefore, $\mathrm{CD}^{+} \mathrm{T}$ cells stimulated by TCR engagement in the absence of $\mathrm{B} 7 / \mathrm{CD} 28$ costimulation exist in a state of clonal anergy that cannot be escaped by entering the cycling pool. These results suggest that CD28 may function independently of cell-cycle progression in the avoidance of anergy.

Interestingly, this form of clonal anergy is associated with the inability to downregulate the cell-cycle inhibitor $\mathrm{p} 27^{\mathrm{kip} 1}$ in response to re-ligation of the TCR (Figure 2p). p2 $7^{\text {kip } 1}$ regulates cell-cycle progression in $\mathrm{T}$ cells by inhibiting the activity of the cyclin-dependent kinase (CDK) responsible for phosphorylation of the retinoblastoma tumor suppressor $(\mathrm{Rb})$, thereby blocking the release of E2F transcription factor and the expression of genes required for the transition from $\mathrm{G} 1$ to $S$ phase $(24,25)$. Productively primed $T$ cells secrete IL-2 in response to TCR reengagement (Figure 1a), which in turn signals the degradation of p27kip1 (ref. 26; Figure $2 \mathrm{p}$, bottom panel, first and second lanes). However, in T cells primed in the presence of CTLA4Ig, p27kip1 levels remained high after TCR re-ligation (Figure $2 \mathrm{p}$, bottom panel, third lane). This represents a defect in downregulation, as both primed and anergic $\mathrm{T}$ cell populations contained comparable, high levels of p2 $7^{\text {kip } 1}$ before restimulation (Figure $2 \mathrm{p}$, top panels). The elevated levels of $\mathrm{p}^{27^{\mathrm{kip}} 1}$ in these anergic T cells is most likely due to inadequate IL-2 production in response to restimulation (Figure $1 \mathrm{~b}$ ), because the addition of exogenous IL-2 resulted in efficient downregulation of p27 kip1 (Figure 2o, fourth lane). This result may explain why exogenous IL-2 is also able to restore the proliferative capacity of anergic $\mathrm{T}$ cells (ref. 27; Figure 2o). Our data do not differentiate whether $\mathrm{p} 27^{\mathrm{kip} 1}$ is playing a causal role in the induction and/or maintenance of anergy in our model or simply acts as an "indicator" of proliferative potential. However, $\mathrm{p} 27^{\mathrm{kip} 1}$ has been recent- ly suggested to be an active "anergy factor" in human $\mathrm{T}$ cell clones rendered unresponsive by TCR engagement in the absence of CD28 costimulation, as it can specifically inhibit IL-2 gene transcription in addition to inhibiting CDK activity (28). Therefore, it is possible that elevated levels of $\mathrm{p} 27^{\text {kip } 1}$ could represent a biochemical mechanism by which clonal anergy is maintained in this model of primary $\mathrm{T}$ cell unresponsiveness and may contribute to the "depth" of tolerance induced by costimulatory blockade.

CD28 costimulation is not sufficient for anergy avoidance by primary $\mathrm{CD}^{+} T$ cells: requirement for cell cycle progression. The results described above suggest that B7/CD28 costimulation is absolutely necessary for anergy avoidance, because even $\mathrm{CD} 4^{+} \mathrm{T}$ cells that achieve multiple rounds of cell division during costimulatory blockade remain refractory to TCR reengagement. However, it remained unclear whether CD28 signals were by themselves sufficient for the avoidance of anergy, or whether other factors may further regulate anergy avoidance in this model. To examine whether cell-cycle progression is an important regulator of anergy avoidance under conditions of sufficient $\mathrm{CD} 28$ costimulatory signals, $\mathrm{CD} 4^{+}$ $\mathrm{T}$ cells primed in the presence of normal B7-mediated costimulation were sorted as above into fractions that had either achieved two cell divisions, or had remained undivided throughout the entire culture period. Under these conditions, the proliferative behavior of the individual $\mathrm{CD}^{+} \mathrm{T}$ cells markedly influenced their secondary responsiveness. Those $\mathrm{CD} 4^{+} \mathrm{T}$ cells that proliferated during the primary stimulus proliferated well in response to restimulation (Figure 2d; Figure 2o, first set of columns). However, $\mathrm{CD}^{+} \mathrm{T}$ cells from the same culture that failed to divide after activation exhibited a marked reduction in their capacity to proliferate in response to restimulation (Figure 2e; Figure 2o, first set of columns). These data confirm the results of our previous studies (4) and are also consistent with experiments in which the inhibition of cell-cycle progression by IL-2/IL-2R blockade (16), butyrate (29), or rapamycin (18) induced anergy in cloned T cells despite the presence of $\mathrm{CD} 28$ costimulation. However, we extend the observations from these studies, which were performed under conditions in which failure of cell cycle progression was induced by exogenous pharmacological agents, to cell-cycle arrest occurring "normally" as part of the observed heterogeneity of $\mathrm{T}$ cell responses. Together, these studies suggest that the secondary responsiveness of $\mathrm{CD}^{+} \mathrm{T}$ cells stimulated in the presence of $\mathrm{CD} 28$ costimulation is dependent on primary cell division.

$\mathrm{CD}^{+}$T cells that fail to proliferate after TCR/CD28-mediated activation are refractory to IL-2. Unlike cells anergized by TCR engagement in the absence of CD28 signals, the $\mathrm{CD}^{+} \mathrm{T}$ cells that failed to proliferate during primary TCR/CD28 stimulation were markedly refractory to subsequent IL-2 stimulation compared with the divided cells derived from the same primary cultures (Figure 2, compare $\mathrm{f}$ and g; Figure 2o, compare open and shad- 


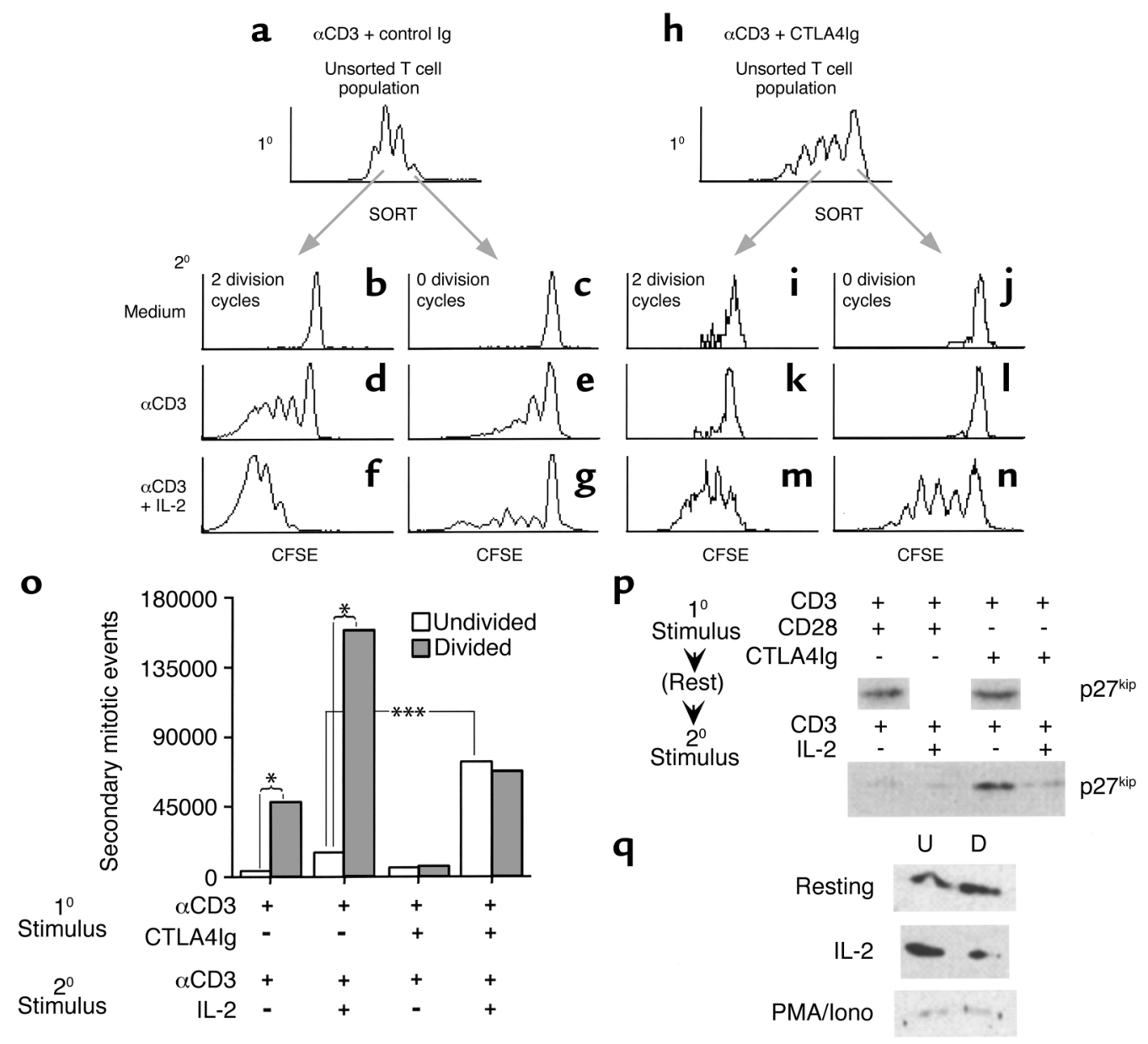

\section{Figure 2}

B7-mediated costimulation and cell division differentially regulate secondary T cell proliferation. (a-o) CFSE-labeled spleen and lymph node cells were stimulated with anti-CD3 in the presence of human $\operatorname{lgG}(\mathbf{a}-\mathbf{g}$; first and second sets of columns in $\mathbf{o})$ or CTLA4Ig (h-n; third and fourth sets of columns in $\mathbf{o}$ ). Cultures were rested for 48 hours, and Thy $1.2^{+}$cells that had divided twice (b, $\mathbf{d}, \mathbf{f}, \mathbf{i}, \mathbf{k}$, and $\mathbf{m}$; shaded bars in $\left.\mathbf{o}\right)$ or had remained undivided ( $\mathbf{c}, \mathbf{e}, \mathbf{g}, \mathbf{j}, \mathbf{I}$, and $\mathbf{n}$; open bars in $\mathbf{o}$ ) following primary stimulation were purified by FACS. The sorted T cells were cultured with irradiated APCs and restimulated with anti-CD3 in the presence or absence of exogenous IL-2; proliferation was assessed 4 days later by flow cytometry. One representative experiment for each condition (a-g and $\mathbf{h}-\mathbf{n})$ is depicted graphically. The mean secondary mitotic events of separate experiments ( $n=2$ [first and second sets of columns] or $n=4$ [third and fourth sets of columns]) are plotted in $\mathbf{o}$. Statistically significant differences were assessed by paired $t$ test and are denoted by brackets: ${ }^{*} P<0.05 ;{ }^{*} * P<0.001$. (p) Lymph node and spleen cells were cultured with anti-CD3 in combination with anti-CD28 antibody (first and second lanes) or CTLA4Ig (third and fourth lanes). The cultures were rested for 24 hours, and the T cells were restimulated with anti-CD3-coated beads for 48 hours in the presence (second and fourth lanes) or absence (first and third lanes) of IL-2. Live cells were harvested after the primary stimulus (top panels) and after the secondary stimulus (bottom panel) by isolation over Ficoll, and lysates were subjected to immunoblot analysis using antibodies against p27kip 1 (top and bottom panels) or actin (data not shown). The results shown are representative of two independent experiments. (q) Primary, CFSE-labeled T cells were primed with anti-CD3 as in $\mathbf{p}$ and rested (top panel), and a portion of the cells were restimulated with either $50 \mathrm{U} / \mathrm{ml} \mathrm{IL}-2$ for 48 hours (middle panel) or PMA/ionomycin (PMA/lono) for 24 hours (bottom panel). The live, CD4 ${ }^{+}$cells were then sorted into fractions that had divided two or more times (right lane, "D"), or had remained undivided during the culture period (left lane, "U"). The cells were lysed, and equal cell equivalents were assessed for $\mathrm{p} 27^{\mathrm{kip} 1}$ content by immunoblot analysis. The data shown are representative of two independent experiments.

ed bars in the second column). The relative inability of the undivided $\mathrm{CD} 4^{+} \mathrm{T}$ cells to utilize IL-2 as a growth factor in this system is associated with an increased level of $\mathrm{p}^{2 \mathrm{kip} 1}$ after IL-2 treatment, compared with the reduced level of $\mathrm{p} 27^{\mathrm{kip} 1}$ observed in the divided $\mathrm{CD}^{+}$ subset (Figure 2q, compare lanes " $U$ " and " $D$ " in the first two panels). This inability to downregulate p27kip1 in response to IL-2 is not due to a lack of IL-2 receptor expression, as these cells exhibit normal levels of IL-2R $\alpha$, IL-2R $\beta$, and IL-2R $\gamma_{c}$ as measured by flow cytometry (4), and other functional consequences of IL-2-mediated signaling, such as STAT5 activation (4) and $\mathrm{Bcl}-2$ upregulation (our unpublished observations), occur normally in these cells. Furthermore, receptorindependent stimulation using PMA and ionomycin led

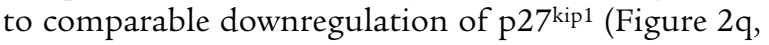
third panel) and proliferation (data not shown; ref. 4) by both the divided and undivided subsets, demonstrating that distal cell-cycle machinery is present and functional in the undivided cells. Together, these data suggest 
that signal transduction pathways leading to cell-cycle progression are specifically uncoupled from the IL-2 receptor in those cells that fail to divide during primary activation. These results also imply that CD28 costimulation is necessary but not sufficient for anergy avoidance by $\mathrm{CD}^{+} \mathrm{T}$ cells, and further emphasize the importance of cell-cycle progression in regulating $\mathrm{T}$ cell differentiation and effector function.

Development of division-arrest-associated IL-2 unresponsiveness is dependent on CTLA-4-B7 interactions during primary stimulation. To further characterize factors in addition to CD28 and cell division that regulate anergy avoidance in $\mathrm{CD} 4^{+} \mathrm{T}$ cells, we focused on the alternate receptor for $\mathrm{B} 7$ on $\mathrm{T}$ cells, CTLA-4. Unlike CD28, CTLA- 4 negatively regulates bulk $\mathrm{T}$ cell responses by inhibiting transcription of the IL-2 gene and impeding clonal expansion $(10,11)$. CTLA-4 has also been shown to be important for the induction of tolerance in several experimental models $(12,14,30)$.

To study how individual $\mathrm{CD}^{+} \mathrm{T}$ cells respond to CTLA-4 signals, we cultured primary, CFSE-labeled spleen cells with polystyrene beads coated with various combinations of anti-CD3, anti-CD28, or anti-CTLA-4 (Figure 3). Used in this fashion (i.e., immobilized on beads) the anti-CTLA- $4 \mathrm{mAb}$ has been shown previously to stimulate the CTLA- 4 receptor and deliver a negative signal to T cells (9). Consistent with this, we found that engagement of TCR and CD28 in the absence of CTLA-4 signals resulted in the proliferation of nearly $80 \%$ of the input $\mathrm{CD}^{+} \mathrm{T}$ cells in the culture, with the average responding precursor generating between 10 and 11 daughter cells (Figure 3a). However, only approximately $45 \%$ of the input $\mathrm{CD}^{+} \mathrm{T}$ cells entered the cycling pool when CTLA-4 was co-crosslinked with TCR and $\mathrm{CD} 28$, and fewer than seven daughter cells were generated by the average responder under these conditions (Figure 3b). Therefore, CTLA- 4 engagement increases the probability that an individual $\mathrm{CD}^{+} \mathrm{T}$ cell will fail entirely to proliferate after primary activation, and it also restricts the potential of those cells that do respond to undergo multiple rounds of cell division.

An unanticipated finding of the experiments described in Figure 2 was that the T cell pool that remains undivided after primary TCR stimulation in the presence of CTLA4Ig was able to respond to restimulation with anti-CD3 plus IL-2, whereas undivided $\mathrm{T}$ cells arising from primary cultures stimulated without CTLA4Ig were refractory to restimulation (Figure 2o, compare second and fourth sets of columns). Because CTLA4Ig in these cultures blocks ligation of both CD28 and CTLA-4, the known regulatory role of CTLA-4 suggested that the development of nonresponsiveness in undivided cells under conditions of TCR stimulation in the presence of B7 ligands might result from CTLA- 4 engagement. To test this possibility, we selectively blocked B7-CTLA-4 interactions during primary stimulation using either whole anti-CTLA-4 antibody, or anti-CTLA-4 Fab fragments. While overt crosslinking of CTLA-4 result- ed in a decrease in the frequency of proliferating $\mathrm{CD}^{+}$ $T$ cells (Figure 3 ), the addition of anti-CTLA- 4 antibody or Fab fragments resulted in a $10 \%$ increase in the frequency of proliferative $\mathrm{CD}^{+} \mathrm{T}$ cells compared with cultures where physiological B7-CTLA-4 interactions were allowed (data not shown). Nonetheless, many $\mathrm{CD}^{+} \mathrm{T}$ cells $(\sim 25 \%)$ still failed to proliferate under these conditions, enabling us to assess the responsiveness of the resultant divided versus undivided $\mathrm{CD} 4^{+} \mathrm{T}$ cells to restimulation with anti-CD3 or IL-2 (Figure 4, a-g).

Similar to the $\mathrm{CD}^{+} \mathrm{T}$ cells that failed to divide in response to soluble anti-CD3 (Figure 2o, first set of columns) or anti-CD3 plus CTLA4Ig (Figure 2o, third set of columns), the undivided $\mathrm{CD}^{+} \mathrm{T}$ cells derived from cultures treated with anti-CTLA-4 antibody proliferated poorly in response to restimulation with antiCD3 (Figure 4e; Figure 4o, third set of columns). Therefore, the addition of anti-CTLA-4 during primary stimulation did not restore the responsiveness of these cells to TCR/CD28 ligation. However, anti-CTLA-4 treatment during primary stimulation was able to restore the responsiveness of the undivided $\mathrm{CD}^{+} \mathrm{T}$ cells to IL-2, as the fraction of $\mathrm{CD} 4^{+} \mathrm{T}$ cells that failed to divide during CTLA-4 blockade exhibited a fivefold greater proliferative response to IL-2 than undivided $\mathrm{CD}^{+} \mathrm{T}$ cells stimulated under conditions that allowed CTLA-4-B7 interactions (Figure 4g; Figure $4 \mathrm{~h}$, compare column sets two and four). Because both anti-CTLA-4 Fab fragments (as shown in Figure 4, a-g) and whole anti-CTLA-4 antibody (Figure 4h) had similar effects in this model, we conclude that the a

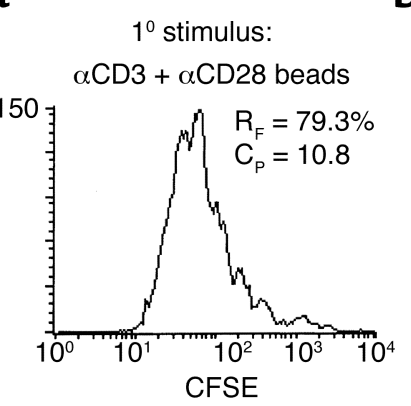

b $\quad 1^{0}$ stimulus: $\alpha \mathrm{CD} 3+\alpha \mathrm{CD} 28$ $+\alpha$ CTLA -4 beads

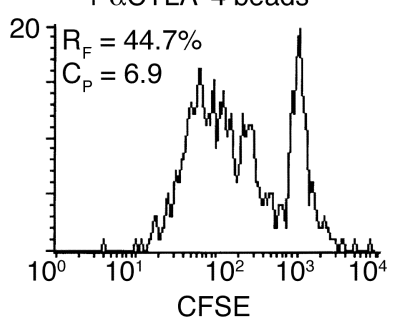

\section{Figure 3}

Regulation of primary T cell division by B7-CTLA-4 interactions. (a) Pooled BALB/c lymph node and spleen cells were labeled with CFSE, and $T$ cells were subjected to co-crosslinking of TCR and CD28 by the addition of polystyrene beads coated with anti-CD3 $(1 \mu \mathrm{g} / \mathrm{ml})$, anti-CD28 $(1 \mu \mathrm{g} / \mathrm{ml})$, and control hamster $\operatorname{lgG}(1 \mu \mathrm{g} / \mathrm{ml})$. (b) In separate cultures, $T$ cells were subjected to co-crosslinking of TCR, CD28, and CTLA-4 by the addition of polystyrene beads coated with anti-CD3 $(1 \mu \mathrm{g} / \mathrm{ml})$, anti-CD28 $(1 \mu \mathrm{g} / \mathrm{ml})$, and anti-CTLA-4 (1 $\mu \mathrm{g} / \mathrm{ml})$. Proliferation of the $\mathrm{CD} 4^{+} \mathrm{T}$ cell subset was assessed by flow cytometry 3 days later. The frequency of precursor T cells that divided in response to stimulus $\left(R_{F}\right)$, and the number of daughter $T$ cells generated by the average responding precursor $T$ cell $\left(C_{P}\right)$, were calculated as described previously $(2,3)$. The data are representative of two separate experiments. 
a

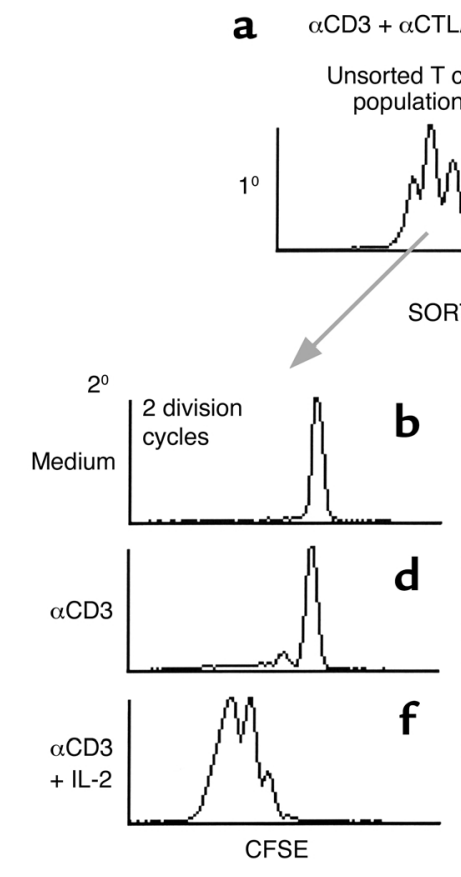

h

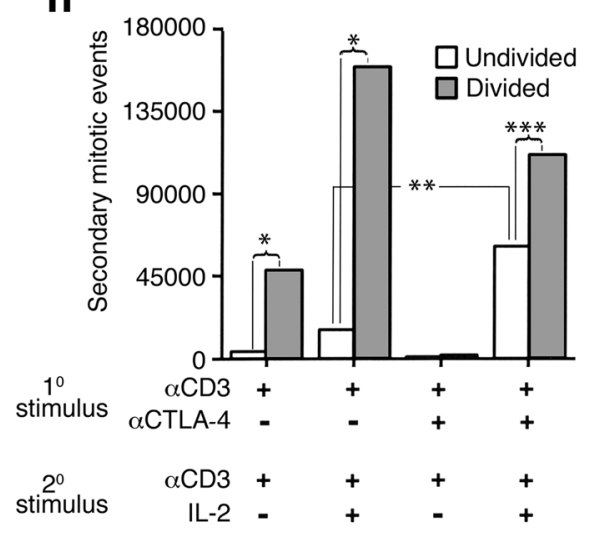

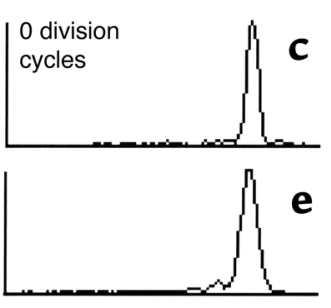
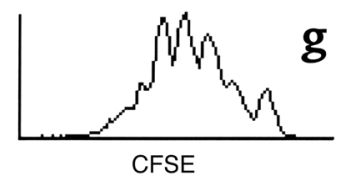

i

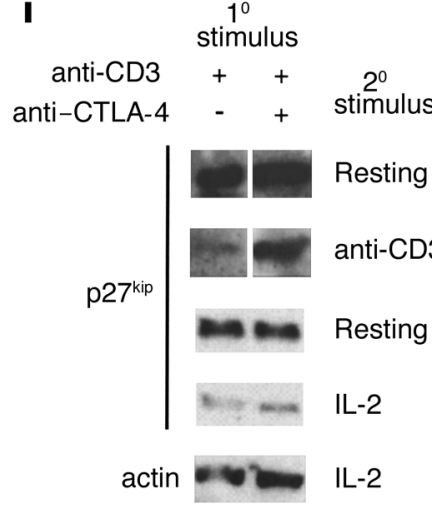

\section{Figure 4}

Regulation of secondary $T$ cell proliferation by CTLA4-mediated signal transduction and cell division during the primary response. (a-h) CFSE-labeled splenocytes were stimulated with anti-CD3 in the presence of hamster IgG (first and second sets of columns in h), or either anti-CTLA-4 antibody $(10 \mu \mathrm{g} / \mathrm{ml} ; n=2)$ or anti-CTLA-4 Fab (20 $\mu \mathrm{g} / \mathrm{ml} ; n=2$; a-g). Experiments using whole and Fab fragments of anti-CTLA4 antibody gave similar results and are shown combined as $n=4$ experiments ( $h$, third and fourth sets of columns). Cultures were rested for 48 hours, and Thy $1.2^{+}$cells that had divided twice $(\mathbf{b}, \mathbf{d}$, and $\mathbf{f}$; shaded bars in $\mathbf{h}$ ) or had remained undivided (c, e, and $\mathbf{g}$; open bars in $\mathbf{h}$ ) following primary stimulation were purified by FACS. The sorted T cells were cultured with irradiated APCs and restimulated with anti-CD3 in the presence or absence of exogenous IL-2, and proliferation was assessed 4 days later by flow cytometry. One representative experiment is depicted graphically in a-g. The mean secondary mitotic events of separate experiments ( $n=4$, as described above) are plotted in $\mathbf{h}$. The data from the control cultures (first and second sets of columns in $\mathbf{h}$ ) are the same as depicted in Figure 1. Statistically significant differences were assessed by paired $t$ test and are denoted by brackets: ${ }^{*} P<0.05$; ${ }^{*} P<0.01 ;{ }^{*}{ }^{*} P<0.001$. (i) Lymph node and spleen cells were cultured with antiCD3 in combination with anti-CD28 (first lane) or anti-CTLA-4 (second lane). The cultures were then rested for 24 hours (first panel), and a portion of the $T$ cells were restimulated with anti-CD3-coated beads (second panel) for 48 hours. In a separate experiment, lymph node and spleen cells were stimulated as above, rested (third panel), and stimulated with IL-2 ( $50 \mathrm{U} / \mathrm{ml}$; fourth panel) for 48 hours. Live cells were isolated over Ficoll, and lysates were subjected to immunoblot analysis using antibodies against p27kip 1 or actin (fifth panel). The results shown are representative of two independent experiments. biologic effects described above result from blockade of CTLA-4-mediated signal transduction, and not through direct signaling through the CTLA-4 receptor. These results suggest that CTLA- 4 signaling regulates the induction of division-arrest-associated anergy at two levels: (a) by increasing the probability that an individual $\mathrm{CD}^{+} \mathrm{T}$ cell will fail to divide after TCR/CD28 stimulation, and (b) by compromising the integrity of IL-2 receptor-mediated signal transduction within the undivided $\mathrm{CD}^{+} \mathrm{T}$ cell pool during restimulation of those cells.

Interestingly, $\mathrm{CD}^{+} \mathrm{T}$ cells that proliferated under conditions of primary CTLA-4/B7 blockade failed to respond to TCR/CD28 restimulation (Figure 4d; Figure $4 \mathrm{~h}$, third set of columns) and instead exhibited an absolute requirement for exogenous IL-2 for secondary proliferation (Figure 4f; Figure $4 \mathrm{~h}$, fourth set of columns). This lack of proliferation in response to secondary TCR engagement was likewise associated with elevated levels of p27kip1 (Figure 4i, second panel, right lane). However, as was the case with prolifera- tion, the capacity of these cells to downregulate p2 $7^{\text {kip } 1}$ was restored by the addition of IL-2 (Figure 4 i, fourth panel). This phenotype is in contrast to $\mathrm{CD}^{+}$ $T$ cells that proliferate under conditions in where CTLA-4 signaling is not blocked, in which case TCR restimulation alone, in the absence of supplemental IL-2, is sufficient to downregulate $\mathrm{p} 27^{\mathrm{kip} 1}$ and induce a proliferative response (Figure 4i, second panel, left lane; Figure $2 \mathrm{~d}$ ). This requirement for IL-2 by $\mathrm{T}$ cells from cultures treated with anti-CTLA-4 may reflect a differential ability of these cells to produce IL-2 upon restimulation compared with $T$ cells from control cultures, or could reflect a different response threshold to IL-2 concentrations. Although unexpected in the context of the many studies that indicate a negative role for CTLA-4 in the development of effector T cells (6), these results are consistent with other studies that show that under some circumstances, CTLA-4 can exert a net positive effect on either primary (31) or secondary (32) T cell responses. It should be noted that one need not invoke a model involving the transduc- 
tion of a positive costimulatory signal through CTLA4 to explain our results in this system. Our findings are consistent with a scenario in which negative signals through CTLA-4 normally oppose the strong TCR-mediated signals transduced by high doses of agonistic anti-CD3, tempering what would otherwise be a supraoptimal response in which IL-2-mediated activation-induced cell death (AICD) $(33,34)$ or receptor desensitization (35) may inhibit subsequent mitogenesis. Consistent with this premise, $\mathrm{CD}^{+} \mathrm{T}$ cells primed in our study with anti-CD3 in the absence of B7-CTLA-4 interactions underwent significantly more cell death upon subsequent TCR crosslinking compared with cells that received physiological CTLA-4 ligation during the primary stimulus (our unpublished observations). Also, reducing the avidity of TCR ligation during primary stimulation in the absence of CTLA-4 signals significantly ameliorated the cell death observed during restimulation (our unpublished observations). Alternatively, inhibition of CTLA-4 signals during high-dose anti-CD3 stimulation may favor the expansion of $\mathrm{T}$ cells with low sensitivity to TCR crosslinking, as has been recently shown in an in vitro human $\mathrm{T}$ cell model, resulting in a postexpansion population with poor sensitivity to TCR re-ligation (32). These two scenarios are not mutually exclusive, and in each case, inhibition of negative CTLA-4 signals during mitogenic stimulation in vitro would result in the development of a $\mathrm{T}$ cell population with a reduced capacity to proliferate upon TCR restimulation.

\section{Discussion}

The data described above demonstrate that two functionally distinct forms of anergy can be resolved in primary $\mathrm{CD}^{+} \mathrm{T}$ cells in vitro (Table 1 ). One form is apparently equivalent to anergy previously defined in long-term $\mathrm{T}$ cell clones (15), in that it results from TCR occupancy in the absence of CD28 costimulation and can be reversed by IL-2 at the time of restimulation. Induction of this form of anergy does not exhibit an absolute requirement for CTLA-4 signaling (36) and, as shown here, cannot be escaped by dividing. The other form of anergy is associated with the failure to proliferate after activation, occurs despite the presence of CD28 costimulatory signals and cannot be reversed by IL-2. This division-arrest-associated form of anergy is controlled by CTLA-4. A biochemical hallmark of both of these forms of anergy is an elevated level of the cell cycle inhibitor p27kip1.

Implications of these two distinct forms of anergy on tolerance in vivo. How might the two forms of anergy described in these in vitro studies be relevant to tolerance induction in vivo? Many modes of tolerance induction in vivo are preceded by some degree of $\mathrm{T}$ cell clonal expansion (37-39). For instance, although CD28 costimulatory blockade significantly reduces the degree of clonal expansion exhibited by antigen-specific T cells in vivo (3, 40 ), some proliferation does occur, and yet tolerance is

achieved. Similarly, tolerance induction by anti-CD40L antibody treatment in vivo does not significantly alter the proliferation of antigen-specific T cells (41); however, this phase of clonal expansion leads to clonal deletion and to the development of regulatory $\mathrm{T}$ cells, not to the formation of a functional effector/memory population (42). The ability of costimulatory blockade to induce anergy even in those $\mathrm{T}$ cells that succeed in proliferating in response to an antigenic signal (Figure 2), together with the crucial role that costimulatory blockade plays in the induction of $\mathrm{T}$ cell apoptosis (43), may explain why this treatment is effective at blocking acute allograft rejection $(44,45)$ and autoimmune pathology $(46,47)$.

Superantigen-induced tolerance in vivo is also preceded by a massive phase of clonal expansion, as well as an equally massive phase of clonal deletion, and is associated with the persistence of a trace population of specific $T$ cells that are unresponsive to subsequent stimulation $(48,49)$. Interestingly, Renno et al. have shown that only a fraction of the superantigen-reactive $T$ cells actually participate in clonal expansion during this response and that essentially all the divided cells are deleted by apoptosis (50). Conversely, those superantigen-reactive cells that fail to proliferate survive the phase of clonal deletion and constitute the singular component of the remaining anergic $T$ cell population. This mode of in vivo tolerance induction is strikingly similar to the division-arrest mode of anergy induction described here. Moreover, both division-arrest anergy (Figure 4) and superantigen-induced anergy (51) are dependent on CTLA-4 signals.

Together with results from previous studies $(4,16,18$, 29), the data presented here suggest that CD28 costimulation is necessary but not sufficient for anergy avoidance in primary $\mathrm{CD}^{+} \mathrm{T}$ cells. To escape anergy induction, a $T$ cell that has received antigenic and costimulatory signals must also successfully progress through the cell cycle. The failure of either of these events leads to two distinct forms of unresponsiveness. A more thorough understanding of how these two modes of anergy might differentially contribute to tolerance induction in various clinical settings such as autoimmunity and transplantation may lead to therapeutic approaches with greater specificity and success.

\section{Table 1}

Functional comparison of two distinct forms of anergy occurring in primary $\mathrm{CD} 4^{+} \mathrm{T}$ cells in vitro

\section{Attribute}

Induced by lack of CD28 costimulatory signals Induced by CTLA-4 signals Dependent on cell division Reversed by IL-2

Associated with elevated levels of $\mathrm{p} 27^{\mathrm{kip} 1}$

$\begin{array}{cc}\begin{array}{c}\text { Clonal anergy } \\ \text { Yes }\end{array} & \text { Division-arrest anergy } \\ & \text { No } \\ \text { No }^{A} & \text { Yes }^{\mathrm{B}} \\ \text { No } & \text { Yes } \\ \text { Yes } & \text { No } \\ \text { Yes } & \text { Yes }\end{array}$

ASee Frauwirth et al. (36). BIL-2-refractory phenotype only; TCR-responsiveness is not affected. 


\section{Acknowledgments}

The authors thank Daniel Mueller, Andrew Caton, and Steven Reiner for helpful discussions. These studies were supported by grants from the NIH (K01DK02771-02 to A.D. Wells; AI-37691, AI-41521, and AI43626 to L.A. Turka). L.A. Turka is an Established Investigator of the American Heart Association.

1. Bucy, R.P., et al. 1994. Heterogeneity of single cell cytokine gene expression in clonal T cell populations. J. Exp. Med. 180:1251-62.

2. Wells, A.D., Gudmundsdottir, H., and Turka, L.A. 1997. Following the fate of individual $\mathrm{T}$ cells throughout activation and clonal expansion. Signals from $\mathrm{T}$ cell receptor and CD28 differentially regulate the induction and duration of a proliferative response. J. Clin. Invest. 100:3173-3183

3. Gudmundsdottir, H., Wells, A.D., and Turka, L.A. 1999. Dynamics and requirements of $\mathrm{T}$ cell clonal expansion in vivo at the single-cell level: effector function is linked to proliferative capacity. J. Immunol. 162:5212-5223.

4. Wells, A.D., Walsh, M.C., Sankaran, D., and Turka, L.A. 2000. T cell effector function and anergy avoidance are quantitatively linked to cell division. J. Immunol 165:2432-2443.

5. Lenschow, D.J., Walunas, T.L., and Bluestone, J.A. 1996. CD28/B7 system of T cell costimulation. Annu. Rev. Immunol. 14:233-258.

6. Oosterwegel, M.A., Greenwald, R.J., Mandelbrot, D.A., Lorsbach, R.B., and Sharpe, A.H. 1999. CTLA-4 and T cell activation. Curr. Opin. Immunol. 11:294-300.

7. June, C.H., Ledbetter, J.A., Gillespie, M.M., Lindsten, T., and Thompson, C.B. 1987. T-cell proliferation involving the CD28 pathway is associated with cyclosporine-resistant interleukin 2 gene expression. Mol. Cell Biol. 7:4472-4481.

8. Harding, F.A., McArthur, J.G., Gross, J.A., Raulet, D.H., and Allison, J.P. 1992. CD28-mediated signalling co-stimulates murine $T$ cells and prevents induction of anergy in T-cell clones. Nature. 356:607-609.

9. Krummel, M.F., and Allison, J.P. 1995. CD28 and CTLA-4 have opposing effects on the response of $\mathrm{T}$ cells to stimulation. J. Exp. Med. 182:459-465.

10. Walunas, T.L., Bakker, C.Y., and Bluestone, J.A. 1996. CTLA-4 ligation blocks CD28-dependent T cell activation. J. Exp. Med. 183:2541-2550.

11. Krummel, M.F., and Allison, J.P. 1996. CTLA-4 Engagement inhibits IL2 accumulation and cell cycle progression upon activation of resting $\mathrm{T}$ cells. J. Exp. Med. 183:2533-2540.

12. Perez, V.L., et al. 1997. Induction of peripheral T cell tolerance in vivo requires CTLA-4 engagement. Immunity. 6:411-417.

13. Brunner, M.C., et al. 1999. CTLA-4-Mediated inhibition of early events of $\mathrm{T}$ cell proliferation. J. Immunol. 162:5813-5820.

14. Greenwald, R.J., Boussiotis, V.A., Lorsbach, R.B., Abbas, A.K., and Sharpe, A.H. 2001. CTLA-4 regulates induction of anergy in vivo. Immunity. 14:145-155.

15. Schwartz, R.H. 1990. A cell culture model for T lymphocyte clonal anergy. Science 248:1349-1356.

16. DeSilva, D.R., Urdahl, K.B., and Jenkins, M.K. 1991. Clonal anergy is induced in vitro by $\mathrm{T}$ cell receptor occupancy in the absence of proliferation. J. Immunol. 147:3261-3267.

17. Wells, A.D., and Turka, L.A. 1998. Individual T cells hold unexpected clues to the nature of anergy and memory. Immunol. Res. 17:261-268.

18. Powell, J.D., Lerner, C.G., and Schwartz, R.H. 1999. Inhibition of cell cycle progression by rapamycin induces $T$ cell clonal anergy even in the presence of costimulation. J. Immunol. 162:2775-2784.

19. Telander, D.G., Malvey, E.N., and Mueller, D.L. 1999. Evidence for repression of IL-2 gene activation in anergic T cells. J. Immunol. 162:1460-1465.

20. Reiner, S.L., and Seder, R.A. 1999. Dealing from the evolutionary pawnshop: how lymphocytes make decisions. Immunity. 11:1-10.

21. Openshaw, P., et al. 1995. Heterogeneity of intracellular cytokine synthesis at the single-cell level in polarized $\mathrm{T}$ helper 1 and $\mathrm{T}$ helper 2 populations. J. Exp. Med. 182:1357-1367.

22. Bird, J.J., et al. 1998. Helper T cell differentiation is controlled by the cell cycle. Immunity 9:229-237.

23. Gett, A.V., and Hodgkin, P.D. 1998. Cell division regulates the T cell cytokine repertoire, revealing a mechanism underlying immune class regulation. Proc. Natl. Acad. Sci. USA. 95:9488-9493.
24. Sherr, C.J. 1996. Cancer cell cycles. Science. 274:1672-1677.

25. Morgan, D.O. 1995. Principles of CDK regulation. Nature. 374:131-134.

26. Nourse, J., et al. 1994. Interleukin-2-mediated elimination of the p27Kip1 cyclin-dependent kinase inhibitor prevented by rapamycin. Nature. 372:570-573.

27. Beverly, B., Kang, S.M., Lenardo, M.J., and Schwartz, R.H. 1992. Reversal of in vitro $\mathrm{T}$ cell clonal anergy by IL-2 stimulation. Int. Immunol. 4:661-671.

28. Boussiotis, V.A., et al. 2000. p27kip1 functions as an anergy factor inhibiting interleukin 2 transcription and clonal expansion of alloreactive human and mouse helper T lymphocytes. Nat. Med. 6:290-297.

29. Gilbert, K.M., and Weigle, W.O. 1993. Th1 cell anergy and blockade in G1a phase of the cell cycle. J. Immunol. 151:1245-1254.

30. Bluestone, J.A. 1997. Is CTLA-4 a master switch for peripheral T cell tolerance? J. Immunol. 158:1989-1993.

31. Wu, Y., Guo, Y., Huang, A., Zheng, P., and Liu, Y. 1997. CTLA-4-B7 interaction is sufficient to costimulate T cell clonal expansion. J. Exp. Med. 185:1327-1335.

32. Anderson, D.E., et al. 2000. Paradoxical inhibition of T-cell function in response to CTLA-4 blockade; heterogeneity within the human T-cell population. Nat. Med. 6:211-214.

33. Russell, J.H., White, C.L., Loh, D.Y., and Meleedy-Rey, P. 1991. Receptorstimulated death pathway is opened by antigen in mature T cells. Proc. Natl. Acad. Sci. USA. 88:2151-2155.

34. Lenardo, M.J. 1991. Interleukin-2 programs mouse alpha beta $\mathrm{T}$ lymphocytes for apoptosis. Nature. 353:858-861.

35. De Mattia, F., et al. 1999. Antigen-experienced T cells undergo a transient phase of unresponsiveness following optimal stimulation. $J$. Immunol. 163:5929-5936.

36. Frauwirth, K.A., Alegre, M.L., and Thompson, C.B. 2000. Induction of T cell anergy in the absence of CTLA-4/B7 interaction. J. Immunol. 164:2987-2993.

37. Kurts, C., Kosaka, H., Carbone, F.R., Miller, J.F., and Heath, W.R. 1997. Class I-restricted cross-presentation of exogenous self-antigens leads to deletion of autoreactive CD8(+) T cells. J. Exp. Med. 186:239-245.

38. Pape, K.A., Mercia, R., Mondino, A., Khoruts, A., and Jenkins, M.K. 1998. Direct evidence that functionally impaired CD4+ T cells persist in vivo following induction of peripheral tolerance. J. Immunol. 160:4719-4729.

39. Adler, A.J., Huang, C.-T., Yochum, G.S., Marsh, D.W., and Pardoll, D.M. 2000. In vivo CD4+ T cell tolerance induction versus priming is independent of the rate and number of cell divisions. J. Immunol. 164:649-655.

40. Kearney, E.R., et al. 1995. Antigen-dependent clonal expansion of a trace population of antigen- specific CD4+ T cells in vivo is dependent on CD28 costimulation and inhibited by CTLA-4. J. Immunol. 155:1032-1036.

41. Kirk, A.D., et al. 1999. Treatment with humanized monoclonal antibody against CD154 prevents acute renal allograft rejection in nonhuman primates. Nat. Med. 5:686-693.

42. Blair, P.J., et al. 2000. CD40 ligand (CD154) triggers a short-term CD4(+) $T$ cell activation response that results in secretion of immunomodulatory cytokines and apoptosis. J. Exp. Med. 191:651-660.

43. Wells, A.D., et al. 1999. Requirement for T-cell apoptosis in the induction of peripheral transplantation tolerance. Nat. Med. 5:1303-1307.

44. Turka, L.A., et al. 1992. T-cell activation by the CD28 ligand B7 is required for cardiac allograft rejection in vivo. Proc. Natl. Acad. Sci. USA. 89:11102-11105.

45. Larsen, C.P., et al. 1996. Long-term acceptance of skin and cardiac allografts after blocking CD40 and CD28 pathways. Nature. 381:434-438.

46. Miller, S.D., et al. 1995. Blockade of CD28/B7-1 interaction prevents epitope spreading and clinical relapses of murine EAE. Immunity. 3:739-745.

47. Grewal, I.S., et al. 1996. Requirement for CD40 ligand in costimulation induction, T cell activation, and experimental allergic encephalomyelitis. Science. 273:1864-1867.

48. Hermann, T., Baschieri, S., Lees, R.K., and MacDonald, H.R. 1992. In vivo responses of CD4+ and CD8+ cells to bacterial superantigens. Eur. J. Immunol. 7:1935-1938.

49. Rellahan, B.L., Jones, L.A., Kruisbeek, A.M., Fry, A.M., and Matis, L.A. 1990. In vivo induction of anergy in peripheral $\mathrm{V}$ beta $8+\mathrm{T}$ cells by staphylococcal enterotoxin B. J. Exp. Med. 172:1091-1100.

50. Renno, T., Hahne, M., and MacDonald, H.R. 1995. Proliferation is a prerequisite for bacterial superantigen-induced $\mathrm{T}$ cell apoptosis in vivo. $J$. Exp. Med. 181:2283-2287.

51. Walunas, T.L., and Bluestone, J.A. 1998. CTLA-4 regulates tolerance induction and $\mathrm{T}$ cell differentiation in vivo. J. Immunol. 160:3855-3860. 